\title{
Dental research waste in design, analysis, and reporting: A scoping review
}

Nikolaos Pandis ${ }^{1}$, Padhraig S. Fleming ${ }^{2}$, Christos Katsaros ${ }^{1}$, John P.A. Ioannidis ${ }^{3}$

1 Department of Orthodontics and Dentofacial Orthopedics, School of Dental Medicine, Medical Faculty, University of Bern, Bern, Switzerland.

2 Barts and The London School of Medicine and Dentistry, Queen Mary University of London, London, United Kingdom.

3 Departments of Medicine, of Epidemiology and Population Health, of Biomedical Data Science, and of Statistics, and Meta-Research Innovation Center at Stanford (METRICS), Stanford University, Stanford, California, USA

Keywords: research waste, study bias, sample size, study analysis, conflict of interest, study reporting

\section{Abstract}

Research waste is highly prevalent across biomedical investigation. We aimed to assess the evidence on the extent of research waste in dental research. We performed a scoping review of empirical evaluations of dental studies assessing the prevalence and impact of limitations in design, conduct, analysis, and reporting of research. PubMed was searched using specific terms to retrieve studies dealing with design, conduct, analysis, and reporting of studies in dentistry, with no year or language restrictions. Of 1807 publications identified from the search and from manual searches, 71 were included in this review. The existing evidence suggests that, although there are improvements over time, substantial deficiencies in all areas (design, conduct, analysis, reporting) were prevalent in dental research publications. Waste in research is a multi-faceted problem without a simple solution. However, an appreciation of optimal research design and execution is a prerequisite and should be underpinned by policies that include appropriate training in research methods and properly aligned incentives.

\section{Introduction}

The key aim of biomedical research is to benefit patients, and to this end the production of results which can be trusted and are useful are an imperative. Ideally, clinical decisions should be based on the totality of the evidence; unusable reports may, therefore, compromise decision-making and ultimately the quality of patient care. The validity and usefulness of research results hinges upon a series of aspects such as the conception, design, and execution of studies; funding; regulation and management; and the 
dissemination of findings. Shortcomings in relation to clinical dental research may imperil the yield from research funding and efforts. The following tenets apply equally to medical and dental research (loannidis 2016) :

- Research should aim to tackle real problems which are important for patients

- Research should be applicable to real-life scenarios (pragmatic)

- Research should follow current design principles and be powered to provide conclusive evidence as to whether an intervention is effective or not

- Research should be feasible and good value for money

- Research methodology should be clear and transparent so that it can be trusted and appropriate to use Adequate funding does not necessarily translate into useful clinical research especially in the presence of biased methodology and lack of transparency in reporting (loannidis et al. 2014). Waste in biomedical research has attracted considerable attention in recent years being thrown into particularly sharp focus in a series of articles published by the Lancet in 2014 (loannidis et al. 2014) with five overarching problems being exposed prompting the formation of the REWARD alliance aiming to reduce waste and increase the value of biomedical research. Funding in biomedical research exceeded 240 billion dollars in 2010 with a significant proportion of this originating in the United States (https://www.researchamerica.org/sites/default/files/2016US_Invest_R\&D_report.pdf). It is important to get the best value from this vast investment.

Complete, transparent trial reporting facilitates accurate assessment of the quality of the study and correct interpretation of the results. Moreover, identification of studies suitable for inclusion in systematic reviews, appraisal of primary studies, and data extraction are expedited by clear reporting. Dental research is not immune to waste-related problems. For example, existing evidence suggests that the methodological and reporting quality of clinical trials within the different fields of oral health are suboptimal (Pandis et al. 2010; Papageorgiou et al. 2019). Furthermore, complete, and transparent trial reporting facilitates accurate assessment of the quality of the study and correct interpretation of the results. Moreover, identification of studies suitable for inclusion in systematic reviews, appraisal of primary studies, and data extraction are expedited by clear reporting. The goal is to make decisions based on the totality of the evidence; therefore, unusable reports compromise the decision-making process and ultimately the quality of the care provided to patients. 
Collective assessment of the breadth, nature, prevalence, and temporal effects of research waste within dentistry is lacking. Such information would assist in better understanding the current situation, effective historic interventions and in informing approaches to optimizing research yield within dentistry. We therefore performed a scoping review to summarize the extent of waste focusing on deficiencies in design, conduct, and analysis, inaccessibility of data and incomplete and unusable reports.

\section{Materials and Methods}

A scoping review of recently published meta-epidemiologic studies dealing with design, conduct, analysis, and reporting of dental studies was performed. We considered eligible all meta-epidemiological studies evaluating the design, conduct, analysis or reporting of dental publications, and excluded non-systematic reviews, editorials, and articles with no empirical data.

PubMed was searched in February 2020 with no year or language restrictions based on a tailored search strategy (Appendix Table 1) supplemented with a hand search.

\section{Data extraction}

The retrieved studies were uploaded on the Rayyan web app for systematic reviews (https://rayyan.qcri.org/) to facilitate study selection. The titles and abstracts of all studies identified and were assessed by one reviewer with ambiguous cases arbitrated with another assessor. Full-text reports of studies which met the inclusion criteria and for which there was insufficient information in the title and/or abstract to make a clear decision were obtained. Data in relation to dental specialty area or journal of publication, date of publication, type and number of included studies and key study findings were extracted by the first author. Any ambiguities were discussed and reconciled with the second author.

\section{RESULTS}

A total of 1,807 titles from PubMed and other sources were identified. After further assessment of the title and abstract, 1,653 studies were excluded as these did not meet the eligibility criteria. Following evaluation of the full text articles, a further 85 studies were excluded resulting in 71 articles being eligible for inclusion in this scoping review. 
The included studies were grouped based on area of methodological or reporting focus: design, conduct, analysis or reporting of dental studies. In view of the large number of included studies identified, the results are tabulated with a short summary per section.

Waste due to inappropriate design, conduct and analysis of research

Sample size

Adequacy of sample size calculations varied among journals, and was better with inclusion of a methodologist/statistician and multicenter setting. Sample size adequacy ranged from $7.3 \%$ to $35.6 \%$ across the different studies. Significant improvements were observed over time from 1992 to 2012 and a priori sample size calculations were more common in interventional studies (Gratsia et al. 2019b) (Figure 1).

\begin{tabular}{|c|c|c|c|c|c|c|c|}
\hline Study & Period & \# Journals & Subject & $\begin{array}{c}\# \\
\text { Paper } \\
\text { s }\end{array}$ & Study & $\begin{array}{c}\text { Adequacy of } \\
\text { sample size (\%) }\end{array}$ & Change over time \\
\hline $\begin{array}{l}\text { (Pandis et al. } \\
2010 \text { ) }\end{array}$ & 2008-2009 & 6 & Oral Health & 95 & RCTs & 50 & Not assessed \\
\hline $\begin{array}{l}\text { (Pandis et al. } \\
\text { 2011) }\end{array}$ & $2009-2010$ & 6 & Oral Health & 396 & all & 7.3 & Not assessed \\
\hline $\begin{array}{l}\text { (Koletsi, Fleming, } \\
\text { et al. 2014) }\end{array}$ & $1992-2012$ & 8 & Oral Health & 413 & RCTs & 29.3 & $\begin{array}{l}\text { Per year and journal range } \\
\text { 1990: } 0.05 \%-1.75 \% \\
\text { 1995: } 0.016 \%-4.91 \% \\
2000: 0.45 \%-12.97 \% \\
2005: 1.28 \%-30.10 \% \\
2010: 3.62 \%-55.4 \%\end{array}$ \\
\hline $\begin{array}{l}\text { (Koletsi, Pandis, } \\
\text { et al. 2014) }\end{array}$ & $1992-2012$ & 8 & Orthodontics & 139 & RCTs & 29.5 & $\begin{array}{l}\text { 2001-2005: } 22 \% \\
\text { 2005-2008: } 27 \% \\
\text { 2009-2012: } 44 \%\end{array}$ \\
\hline $\begin{array}{l}\text { (Saltaji et al. } \\
\text { 2017) }\end{array}$ & $1955-2013$ & na\# & Oral Health & 540 & RCTs & 17.6 & $\begin{array}{l}\text { 20.9\% average } \\
<1990: 5.5 \% \\
2007-2013: 44.3 \%\end{array}$ \\
\hline $\begin{array}{l}\text { (Gratsia et al. } \\
\text { 2019b) }\end{array}$ & $2015-2018$ & 4 & Orthodontics & 654 & All & 35.6 & No difference \\
\hline
\end{tabular}




\begin{tabular}{|l|c|c|c|c|l|l|l|}
\hline (Dumbrigue, E.C. & & & Prosthodontics & & & Not assessed \\
Dumbrigue, et al. & & & & & & \\
2019) & $2008-2017$ & 2 & & RCTs & $17 \%$ & \\
\hline
\end{tabular}

\#na=not available

Table 1. Summary of studies on sample size calculations.

Randomization and blinding

Despite improvements over time (Dumbrigue, D.C. Dumbrigue, et al. 2019), specific issues included use of inappropriate and/or unclear methods of randomization and lack of blinding (Table 2). Adequacy of randomization ranged from $9 \%$ to $68 \%$ (Appendix Figure 1) and adequacy of blinding ranged from $12 \%$ to $70 \%$ (Appendix Figure 2).

\begin{tabular}{|c|c|c|c|c|c|c|c|}
\hline Study & Period & \# Journals & Subject & \# Papers & Study & Randomization adequacy (\%) & Blinding adequacy (\%) \\
\hline $\begin{array}{l}\text { (Dumbrigue et al. } \\
\text { 2001) }\end{array}$ & 1988-1997 & 3 & Prosthodontics & 62 & RCTs & $47 \%$ & $40 \%$ \\
\hline $\begin{array}{l}\text { (Dumbrigue et al. } \\
2006)\end{array}$ & $1991-2000$ & 17 & $\begin{array}{l}\text { Implant } \\
\text { Dentistry }\end{array}$ & 43 & RCTs & $51 \%$ & $12 \%$ \\
\hline (Ferreira et al. 2011) & 2002-2007 & 40 & $\begin{array}{l}\text { Oral Health (only } \\
\text { LILACS) }\end{array}$ & 78 & RCTs & $9 \%$ & $33.3 \%$ \\
\hline (Koletsi et al. 2012a) & $1979-2011$ & 5 & Oral Health & 222 & RCTs & $39.6 \%$ & not assessed \\
\hline (Koletsi et al. 2012b) & $1979-2011$ & 7 & Orthodontics & 112 & RCTs & $29 \%$ & not assessed \\
\hline $\begin{array}{l}\text { (Papageorgiou et al. } \\
2015 \text { ) }\end{array}$ & $2007-2012$ & 9 & $\begin{array}{l}\text { Implant } \\
\text { Dentistry }\end{array}$ & 147 & RCTs & $40 \%$ & $23 \%$ \\
\hline (Elangovan et al. 2016) & 2013 & 17 & $\begin{array}{l}\text { Periodontal } \\
\text { regeneration }\end{array}$ & 17 & RCTs & $47 \%$ & $70 \%$ \\
\hline (Saltaji et al. 2017) & $1955-2013$ & na\# & Oral health & 540 & RCTs & $\begin{array}{l}\text { Sequence generation: } 32 \% \text { average } \\
\text { Allocation concealment: } 14.1 \% \\
\text { average } \\
\text { sequence generation: } \\
<1990: 11.8 \%, 2007-2013: 51.4 \% \\
\text { allocation concealment: } \\
<1990: 4.7 \%, 2007-2013: 27.8 \%\end{array}$ & $\begin{array}{l}\text { Participants: 71.5\% average } \\
<1990: 65.3 \%, 2007-2013: \\
78.6 \% \\
\text { Assessors: } 59.5 \% \\
<1990: 61.4 \%, 2007-2013: \\
62.1 \%\end{array}$ \\
\hline $\begin{array}{l}\text { (Dumbrigue, et al. } \\
\text { 2019) }\end{array}$ & $2008-2017$ & 3 & Prosthodontics & 96 & RCTs & $\begin{array}{l}\text { 1988-1997: 47\% } \\
\text { 2008-2017: } 68 \%\end{array}$ & $\begin{array}{l}\text { 1988-1997: 40\% } \\
\text { 2008-2017: 50\% }\end{array}$ \\
\hline $\begin{array}{l}\text { (Abdulraheem and } \\
\text { Bondemark 2019) }\end{array}$ & $2012-2018$ & 10 & Orthodontics & 203 & RCTs & Not assessed & $\begin{array}{l}\text { Outcome assessor: } 40.4 \% \\
\text { Single blind: } 15.3 \% \\
\text { Double blind: } 2.5 \%\end{array}$ \\
\hline
\end{tabular}




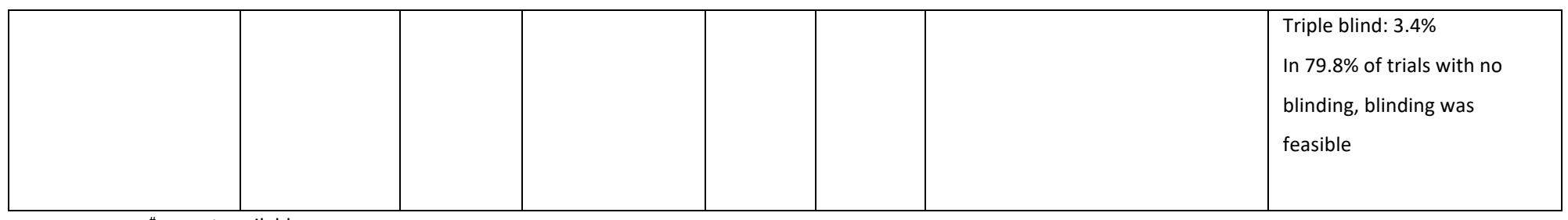

"na=not available

Table 2. Summary of studies on randomization and blinding

Subgroup analyses, testing against baseline, confounding, clustering, and other misuses of statistics

Multiple comparisons via subgroup analyses, testing, and interpreting results from within-group comparisons instead of between group comparisons, lack of adjustments for confounding and clustering either and/or at design or analysis stage were common in dental publications (Table 3). Specifically, in an analysis of studies published in leading journals across five specialty areas over a 4-year period, 40\% incorporated multiple comparisons. The prevalence of multiple comparisons in oral health research ranged from $25.8 \%$ to $42.7 \%$ and the median number of multiple comparisons was 25 in orthodontics and periodontics. Testing against baseline varied across journals and specialties. Interpretation based on change from baseline ranged between $18 \%-56 \%$ again varying across journals and specialties. Ignoring confounding in observational studies was also prevalent (17\% to $21 \%$ ); and ignoring clustering was even more prevalent but also highly variable ( $25 \%$ to $96 \%$ ).

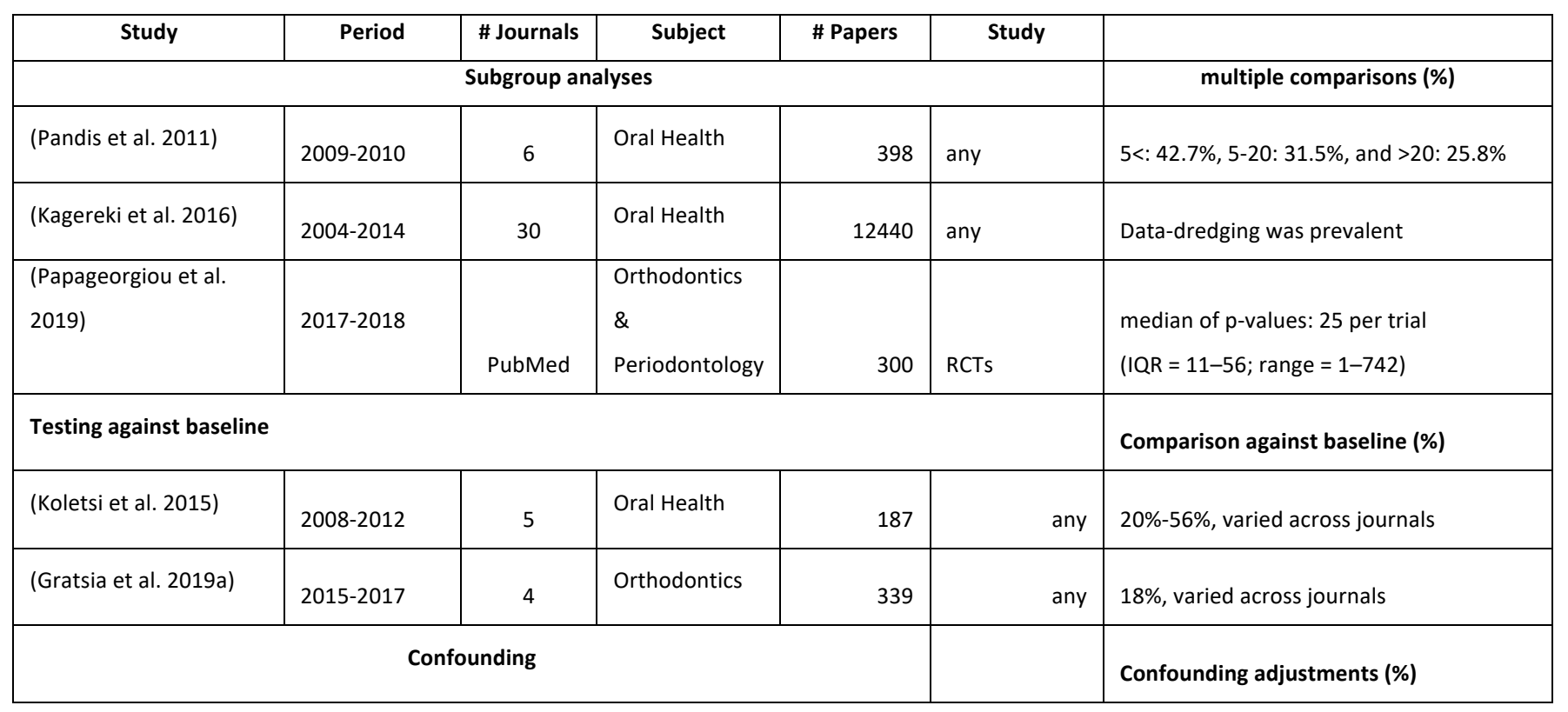




\begin{tabular}{|c|c|c|c|c|c|c|}
\hline (Spanou et al. 2015) & 2010-2014 & 4 & Orthodontic & 426 & $\begin{array}{l}\text { Non- } \\
\text { randomised }\end{array}$ & $17 \%$, varied across journals \\
\hline \multicolumn{6}{|c|}{ Clustering } & Account for clustering $9 \%$ ) \\
\hline (Fleming et al. 2013) & 2009-2012 & 5 & Oral Health & 559 & any & $40 \%$, varied across journals \\
\hline
\end{tabular}

Table 3. Summary of studies on subgroup analyses, testing against baseline, confounding, and clustering

Other misuses of statistics included incorrect use of ordinal data such as pain scores as normally distributed and violation of analysis requirements leading to incorrect application in descriptive statistics (e.g. use of standard deviation instead of interquartile range), correlation, ANOVA, regression analysis and $\chi^{2}$ testing (Kim et al. 2011). In a systematic review assessing outcomes of implant studies it was reported that in $98.6 \%$ of the cases the unit of analysis was the implant; however, it is unclear how many of the included studies involved single or multiple implants per patient (Needleman et al. 2012).

\section{Missing data}

Description was not consistent and might pertain to reporting as to whether all patients were accounted for (Dumbrigue et al. 2006), reporting of analysis approach such as complete case (Lieber et al. 2020) , per-protocol (PP) or intention-to-treat (ITT). Missing data were accounted for in $17 \%-98 \%$ of dental studies (Table 4); however, terminology was also often used incorrectly.

\begin{tabular}{|c|c|c|c|c|c|c|}
\hline study & period & \# journals & subject & \# papers & study & $\begin{array}{l}\text { Accounting for Missing data } \\
\text { (\%) }\end{array}$ \\
\hline (Dumbrigue et al. 2006) & $1991-2000$ & 3 & Implant Dentistry & 43 & RCTs & $98 \%$ \\
\hline (Pandis et al. 2010) & 2009 & 6 & Oral Health & 95 & RCTs & $\begin{array}{l}\text { 17\% ITT reported or flow } \\
\text { diagram }\end{array}$ \\
\hline (Papageorgiou et al. 2015) & $2007-2012$ & 9 & Implant Dentistry & 147 & RCTs & $93 \%$ \\
\hline $\begin{array}{l}\text { (Bondemark and } \\
\text { Abdulraheem 2018) }\end{array}$ & 2013-2017 & 5 & Orthodontics & 90 & RCTs & $\begin{array}{l}61.1 \% \text { PP } \\
18.9 \% \text { ITT in the text } \\
6 / 90 \text { correct ITT, } 8 / 90 \\
\text { incorrect ITT implementation }\end{array}$ \\
\hline
\end{tabular}




\begin{tabular}{|c|c|c|c|c|c|c|}
\hline & & & & & & $\begin{array}{l}3 / 90 \text { reported ITT when PP } \\
\text { was actually used } \\
20 \% \text { no dropout reporting }\end{array}$ \\
\hline $\begin{array}{l}\text { (Dumbrigue, D.C. } \\
\text { Dumbrigue, et al. 2019) }\end{array}$ & $2008-2017$ & 3 & Prosthodontics & 96 & RCTs & $85 \%$ \\
\hline (Lieber et al. 2020) & 2015-2018 & na\# & Implant Dentistry & 137 & RCTs & $45 \%$ Complete Case analysis \\
\hline
\end{tabular}

"na=not available

Table 4. Summary of studies on missing data.

Conflict of interest (COI)

Reporting of conflict of interest (COI) and funding in dentistry was uncommon and inconsistent (COI: 8\%-39\%; Funding: 22.5\%-78\%) (Table 5). There was evidence that reporting of funding sources and COI had increased over time. The association between industry funding and direction of results was not consistent.

\begin{tabular}{|c|c|c|}
\hline study & Characteristics & Funding/COI reporting \\
\hline (Popelut et al. 2010) & $\begin{array}{l}\text { Period: 1993-2008 } \\
\text { Publication type: } 5 \text { systematic reviews } \\
\text { Subject: Implant Dentistry } \\
\mathrm{N}=38 \\
\text { Study type: any clinical study }\end{array}$ & $\begin{array}{l}63 \% \text { Reported funding source } \\
5 \% \text { reported COI }\end{array}$ \\
\hline (Lee 2011) & $\begin{array}{l}\text { Period: } 1998,2003,2008 \\
\text { Publication type: } 3 \text { journals } \\
\text { Subject: Prosthodontics } \\
\mathrm{N}=998 \\
\text { Study type: any }\end{array}$ & 22.5\% Reported funding \\
\hline (Yuan et al. 2011) & $\begin{array}{l}\text { Period: } 2005-2009 \\
\text { Publication type: } 5 \text { journals } \\
\text { Subject: Implant dentistry } \\
\mathrm{N}=1226 \\
\text { Study type: any }\end{array}$ & $\begin{array}{l}\text { No association between positive results industry-funded studies versus } \\
\text { studies funded by other sources and positive outcomes was identifies }\end{array}$ \\
\hline (Fleming et al. 2012) & $\begin{array}{l}\text { Period: } 2006-2011 \\
\text { Publication type: } 4 \text { journals } \\
\text { Subject: Orthodontics } \\
\text { N= } 117 \text { abstracts } \\
\text { Study type: } R C T s\end{array}$ & Sources of funding were not reported in any of the identified abstracts \\
\hline
\end{tabular}




\begin{tabular}{|c|c|c|}
\hline $\begin{array}{l}\text { (Brignardello-Petersen et al. } \\
\text { 2013) }\end{array}$ & $\begin{array}{l}\text { Period: } 2010-2012 \\
\text { Publication type: } 10 \text { journals } \\
\text { Subject: Oral Health } \\
\mathrm{N}=135 \\
\text { Study type: RCTs }\end{array}$ & $\begin{array}{l}37.03 \% \text { source of funding classified as for profit } \\
40.74 \% \text { funded by not-for-profit organization } \\
22.22 \% \text { not reported any source of funding } \\
\text { Reporting of COI was more common in Periodontics }\end{array}$ \\
\hline (Beyari, Hak, et al. 2014) & $\begin{array}{l}\text { Period: } 2011-2012 \\
\text { Publication type: } 6 \text { journals } \\
\text { Subject: Oral Health } \\
\mathrm{N}=66 \\
\text { Study type: } \mathrm{RCTs}\end{array}$ & $\begin{array}{l}76 \% \text { funding and } \mathrm{COI} \\
39 \% \mathrm{COI}\end{array}$ \\
\hline (Alamri and Alharbi 2018) & $\begin{array}{l}\text { Period: 2012-2017 } \\
\text { Publication type: } 2 \\
\text { Subject: Endodontics } \\
\mathrm{N}=109 \\
\text { Study type: RCTs }\end{array}$ & $35 \%$ funding sources \\
\hline (Beyari, Strain, et al. 2014) & $\begin{array}{l}\text { Period: } 2000-2012 \\
\text { Publication type: na } \\
\text { Subject: Oral Health } \\
\mathrm{N}=129 \\
\text { Study type: Meta-analyses }\end{array}$ & $\begin{array}{l}\text { Funding } 38.8 \% \text {. } \\
\text { COI } 8 \% \text {. }\end{array}$ \\
\hline (Chen et al. 2018) & $\begin{array}{l}\text { Period: } 2001-2007,2010-2016 \\
\text { Publication type: } 6 \text { high impact journals } \\
\text { Subject: Prosthetic dentistry } \\
\mathrm{N}=131 \\
\text { Study type: } \mathrm{RCTs}\end{array}$ & $\begin{array}{l}\text { Comparisons between pre-CONSORT and post-CONSORT period } \\
\text { Funding was not reported in both periods }\end{array}$ \\
\hline (Dos Santos et al. 2019) & $\begin{array}{l}\text { Period: } 1996-2016 \\
\text { Publication type: na } \\
\text { Subject: Implant Dentistry } \\
\mathrm{N}=102 \\
\text { Study type: RCTs }\end{array}$ & $\begin{array}{l}\text { 48\% sponsored } \\
\text { No evidence of association between sponsorship status and study } \\
\text { results }\end{array}$ \\
\hline (Lee and Chuang 2020) & $\begin{array}{l}\text { Period: } 2017 \\
\text { Publication type: na } \\
\text { Subject: Oral Health } \\
\mathrm{N}=\text { na } \\
\text { Study type: Open Payments database } \\
\text { of the US Centers for Medicare \& } \\
\text { Medicaid Services }\end{array}$ & $\begin{array}{l}321,627 \text { industry payments totaling } \$ 110,750,601 \\
\text { orthodontists }(61.8 \%) \text {, oral and maxillofacial surgeons }(55.7 \%) \text {, and } \\
\text { periodontists }(54.6 \%) \text {. Most dentists in this study received less than } \\
\$ 200 \text {; however, the distribution of payments was skewed by a few top } \\
\text { earners. }\end{array}$ \\
\hline
\end{tabular}

Table 5. Summary of meta-epidemiological studies relating to conflict of interest in dental research. 
Waste due to inaccessible research, publication, and other reporting biases

From the included studies (Appendix Table 2) it is evident that publication based on statistical significance was prevalent. Specifically, reporting of significant results ranged from $75 \%-97 \%$. Written publication of conference abstracts was relatively low (27\%-55\%). Discrepancies between registry entries and published papers were prevalent with no material improvements over time and with selective outcome reporting estimated at $2-45 \%$. Such practices can compromise healthcare decision making, as key elements of trial data may be missing, and high-quality studies may be inaccessible.

Study registration, protocol, and data sharing

Despite improvements over time, study registration, protocol availability prior to the study commencement and data sharing were quite limited (Appendix Table 3). Registration ranged between $0 \%-76 \%$ and was most often retrospective, while data sharing was scarce.

\section{Waste due to incomplete and unusable reports}

Despite wide adoption of reporting guidelines and improvements over time, reporting has remained suboptimal (Appendix Table 4). Common areas of poor reporting included randomization, sample size calculation, reporting of estimates and measures of precision (confidence intervals). Overall, quality scores ranged from $58.6 \%-87.3 \%$ and varied across journals and specialty fields.

\section{Discussion}

Research waste has implications in clinical practice as scientific evidence forms the foundation of healthcare recommendations. Biased, incomplete, selective, and inaccurate communication of the evidence can result in espousal of ineffective or even potentially harmful therapies. Biased evidence can also be exploited by the industry particularly as dental products may be promoted with aggressive marketing despite a lack of underpinning scientific evidence (Fleming et al. 2012; Livas et al. 2014 ). Based on this scoping review, although there are improvements over time, significant deficiencies in all areas (design, conduct, analysis, reporting) within dental publications persist, risking waste in dental research. 
Design

In terms of design, issues with sample calculation, randomization and blinding remain prevalent within dentistry. Sample calculations contribute to our confidence in the study results and safeguard against inconclusive studies as well as protecting patients' rights. There are ongoing problems concerning both the inclusion of a calculation, the presentation of sufficient information to allow replication, and the accuracy of the calculations themselves. Adequacy of sample size calculations varied among journals but did improve over time with a priori sample size calculations more common in interventional studies (Gratsia et al. 2019). Adequacy of randomization and blinding, which contribute to the validity of the results, varied with positive associations with publication year, number of authors, multicenter setting and involvement of a methodologist (Koletsi et al. 2012a). Saltaji et al (Saltaji et al. 2017) reported that the randomization and blinding implementation in dental trials overall improved significantly over time (1955-2013). Improvements were also reported in orthodontics (Koletsi et al. 2017) and over 30 years in prosthodontics (Dumbrigue, D.C. Dumbrigue, et al. 2019). Both of these design issues are integral to rigorous clinical trials and meaningful, trustworthy findings

\section{Analysis}

Subgroup comparisons and data dredging are associated with false positive results and selective reporting. These issues were prevalent in the dental literature with over $25 \%$ of publications having more than 20 subgroup comparisons (Pandis et al. 2011; Kagereki et al. 2016) with no evidence of improvement over time (Papageorgiou et al. 2019). Interpretation of study results based on withingroup change from baseline comparisons, potentially misleading and with a higher risk of false positive results (Bland and Altman 2011), was common, varied by journal, was strongly associated with statistically significant results and less likely in interventional studies (Koletsi et al. 2015; Gratsia et al. 2019a). Clustering is a common finding in oral health and results in loss of power due to the decrease of the effective sample size and, when ignored, it can lead to smaller $p$-values and significant results which may not be genuine (Campbell et al. 2012). It was common for clustering effects to be ignored and journal type was a significant predictor for accounting for clustering effects (Koletsi et al. 2012c; Fleming et al. 2013)

Missing data may bias treatment results with a clinical trial by leading to unbalanced groups thus producing "unfair" treatment comparisons. Handling of missing data was suboptimal with complete case analysis being more common, whereas mention of ITT (17\%) and correct application of ITT analysis (6\%) 
were less common (Bondemark and Abdulraheem 2018). It seems that there is confusion and inconsistent use of intention-to-treat analysis and a failure to recognize the effect of mechanism of missingness on the further handling of missing observations.

Reporting of conflict of interest and funding

A conflict of interest occurs when professional judgment about a primary interest is overly influenced by a secondary interest such as financial gain. RCTs in which authors have some type of COI are more likely to support the intervention being assessed (Popelut et al. 2010; Brignardello-Petersen et al. 2013), although this pattern was not consistent across all studies (Yuan et al. 2011; Dos Santos et al. 2019). Despite the attempts of the International Committee of Medical Journal Editors (ICMJE) to promote uniform and transparent reporting of conflicts of interest, journal adoption remains suboptimal (Holden and Spallek 2018).

The proportion of studies that reported funding remained relatively constant over the years (Lee 2011). There is evidence that reporting of COI has improved over time with COI statement prior to 2009 in $25 \%$ of the papers and vs 54.1\% of papers since 2009 (Beyari, Strain, et al. 2014). Furthermore, speakers at dental conferences are often sponsored by companies, with full disclosure of the company-speaker relationship to the audience being sporadic. In 2017, leading orthodontic companies ORMCO and Align technologies paid over $\$ 4.5$ million to the ten top highest paid opinion leaders (https://openpaymentsdata.cms.gov/).

Reporting bias and trial registration Reporting bias is prevalent in the dental literature with little improvement over time (Scholey and Harrison 2005; Koletsi et al. 2009; Livas et al. 2014; Smaïl-Faugeron et al. 2014; Fang Hua et al. 2016; Rushing et al. 2018). The vast majority of published orthodontic articles (88\%) showed significant results (Koletsi et al. 2009). A similar range of statistically significant results was found in publications within different dental journals (Polychronopoulou et al. 2010). Interventional studies seem to have fewer statistically significant results compared to other designs (Papageorgiou et al. 2019). This practice can bias decision making as synthesis is based on a subsample of the existing evidence. Proposed remedies to reduce reporting bias include trial registration, availability of the study protocol and ultimately access to the raw data. Inconsistent outcome reporting has prompted the development of core outcome sets (COS) within different disciplines with over 300 now in existence (Gargon et al. 2018) with recent 
publication of an orthodontic COS (Tsichlaki et al., 2020). This approach is also likely to better focus and prioritize interventional studies. Availability of individual patient data can reduce waste by providing transparency and easier and more reliable evaluation of the results, given that errors do occur (Nuijten et al. 2016). Prospective trial registration in the dental field is not common and neither is the availability of the datasets (Smaïl-Faugeron et al. 2015; Papageorgiou et al. 2017; Papageorgiou et al. 2018; Koufatzidou et al. 2019) although efforts to address this are being promoted (Koletsi et al. 2017)

Over the years there is an increase in the percentage of protocols of randomized trials which included a prior citation of randomized trials, systematic reviews, or both. However, improvements are still required to reduce research waste. To the best of our knowledge no relevant empirical evidence is available in oral health.

Despite the plethora of reporting guidelines available in dentistry, suboptimal reporting is prevalent, although improving with time (Sarkis-Onofre et al. 2017). Optimal reporting continues to be impeded by limited endorsement of reporting guidelines within dental journals (Hua et al. 2016). Methods of randomization, sample size calculation and elements of precision are often not reported or inadequately reported in high-impact dental journals indicating that these issues may pervade a range of journals and specialty areas (Pandis et al. 2010; Gratsia et al. 2019a). Reporting is also problematic at the systematic review level resulting in lack of reproducibility of search strategies and in relation to selection of primary studies (Kiriakou et al. 2013). Efforts to enhance the reporting of clinical trials have intensified in recent years with active editorial involvement showing promise in improving compliance with accepted guidelines (Koletsi et al. 2017). The American Journal of Orthodontics and Dentofacial Orthopedics has, for example, successfully adopted an active CONSORT implementation and inclusion of subheadings in order to improve reporting of submitted and published RCTs (Koletsi et al. 2017).

This study is not without limitations. Given the amount of data and the scoping review context this study cannot be considered comprehensive but rather provides in a systematic way an initial view on waste in dental research. Reporting of changes over time was not feasible for all areas covered, either because such information was not available or due to inconsistent definitions and inconsistent reporting within the individual studies. 


\section{Recommendations for Dentistry}

Initiatives such as COMET (http://www.comet-initiative.org/), EQUATOR (Network.

https://www.equator-network.org/), James Lind Alliance Priority Setting Partnerships

(https://www.jla.nihr.ac.uk/priority-setting-partnerships/), Cochrane (

https://oralhealth.cochrane.org/about-us/partnerships-and-funders/global-evidence-ecosystem-oral-

health-geeoh) and REWARD Alliance (https://www.rewardalliance.net/) have directly or indirectly targeted reduction in research waste. In addition, efforts directed at educating researchers and practitioners are a step in the right direction ( https://www.ajodo.org/content/statresearchdesign). Despite those efforts, a significant amount of cohesive work is required to consistently promote more useful dental research. No single or simple solution exists; however, pivotal steps include prioritization of key research themes; improved alignment between patients' needs, scientific progress and academic or career advancement; patient involvement in research design; and concerted focus on early years training of clinicians on research design, methodology and execution. The latter is particularly important as, although clinicians have a positive attitude towards evidence-based dentistry, they claim poor understanding and more importantly often confusion due to the conflicting findings (Madhavji et al. 2011). Moreover, university based studies satisfying thesis and graduation requirements can contribute to waste if not rigorously conducted and are not reported (Nieminen et al. 2007) Finally, incentives in the funding and academic environment should be aligned with these desirable improvements in research practices (Rice et al. 2020)

\section{Acknowledgments}

Funding for this study: None

Conflicts of interest: None

Nikolaos Pandis: Contributed to conception, design, data acquisition, analysis and interpretation, drafted and critically revised the manuscript

Padhraig Fleming: Contributed to conception, design, data acquisition and interpretation, drafted and critically revised the manuscript

Christos Katsaros: Contributed to conception, design, interpretation, drafted and critically revised the manuscript 
John loannidis: Contributed to conception, design, interpretation, drafted and critically revised the manuscript

All authors gave their final approval and agree to be accountable for all aspects of the work.

Figure legend

Figure 1. Flow diagram of study selection

\section{References}

2016US_Invest_R\&D_report.pdf. [accessed 2018 Sep 23].

https://www.researchamerica.org/sites/default/files/2016US_Invest_R\&D_report.pdf.

Abdulraheem S, Bondemark L. 2019. The reporting of blinding in orthodontic randomized controlled trials: where do we stand? Eur J Orthod. 41(1):54-58. doi:10.1093/ejo/cjy021.

Alamri HM, Alharbi F. 2018. Quality Assessment of Randomized Clinical Trials Reporting in Endodontic Journals: An Observational Study from 2012 to 2017. J Endod. 44(8):1246-1250.

doi:10.1016/j.joen.2018.05.011.

Beyari MM, Hak A, Li CS, Lamfon HA. 2014. Conflict of interest reporting in dentistry randomized controlled trials: a systematic review. J Evid-Based Dent Pract. 14(4):158-64.

Beyari MM, Strain D, Li CS, Lamfon HA. 2014. Conflict of interest reporting in dentistry meta-analyses: A systematic review. J Clin Exp Dent. 6(3):e280-5.

Bland JM, Altman DG. 2011. Comparisons against baseline within randomised groups are often used and can be highly misleading. Trials. 12:264. doi:10.1186/1745-6215-12-264.

Bondemark L, Abdulraheem S. 2018. Intention to treat (ITT) analysis as reported in orthodontic randomized controlled trials-evaluations of methodology and recommendations for the accurate use of ITT analysis and handling dropouts. Eur J Orthod. 40(4):409-413. doi:10.1093/ejo/cjx084.

Brignardello-Petersen R, Carrasco-Labra A, Yanine N, Ulloa C, Araya I, Pintor F, Villanueva J, CornejoOvalle M. 2013. Positive association between conflicts of interest and reporting of positive results in randomized clinical trials in dentistry. J Am Dent Assoc 1939. 144(10):1165-70.

Campbell MK, Piaggio G, Elbourne DR, Altman DG, CONSORT Group. 2012. Consort 2010 statement: extension to cluster randomised trials. BMJ. 345:e5661. 
Chen J, Li Z, Liu B, Gan X, Li C, Yu H. 2018. Quality improvement in randomized controlled trial abstracts in prosthodontics since the publication of CONSORT guideline for abstracts: a systematic review. J Dent.:23-29.

Dos Santos MBF, Agostini BA, de Moraes RR, Schwendicke F, Sarkis-Onofre R. 2019. Industry sponsorship bias in clinical trials in implant dentistry: Systematic review and meta-regression. J Clin Periodontol. 46(4):510-519.

Dumbrigue HB, Al-Bayat MI, Ng CCH, Wakefield CW. 2006. Assessment of bias in methodology for randomized controlled trials published on implant dentistry. J Prosthodont Off J Am Coll Prosthodont. 15(4):257-63.

Dumbrigue HB, Dumbrigue DC, Dumbrigue EC, Chingbingyong MI. 2019. Bias: A 30-Year Review of Randomized Controlled Trials Published in Three Prosthodontic Journals. J Prosthodont. 28(6):656-658.

Dumbrigue HB, Dumbrigue EC, Dumbrigue DC, Chingbingyong MI. 2019. Reporting of Sample Size Parameters in Randomized Controlled Trials Published in Prosthodontic Journals. J Prosthodont. 28(2):159-162.

Dumbrigue HB, Jones JS, Esquivel JF. 2001. Control of bias in randomized controlled trials published in prosthodontic journals. J Prosthet Dent. 86(6):592-6.

Elangovan S, Prakasam S, Gajendrareddy P, Allareddy V. 2016. A Risk of Bias Assessment of Randomized Controlled Trials (RCTs) on Periodontal Regeneration Published in 2013. J Evid-Based Dent Pract. 16(1):30-40.

Ferreira CA, Loureiro CAS, Saconato H, Atallah AN. 2011. Assessing the risk of bias in randomized controlled trials in the field of dentistry indexed in the Lilacs (Literatura Latino-Americana e do Caribe em Ciências da Saúde) database. Sao Paulo Med J Rev Paul Med. 129(2):85-93.

Fleming PS, Buckley N, Seehra J, Polychronopoulou A, Pandis N. 2012. Reporting quality of abstracts of randomized controlled trials published in leading orthodontic journals from 2006 to 2011. Am J Orthod Dentofacial Orthop. 142(4):451-8.

Fleming PS, Koletsi D, Polychronopoulou A, Eliades T, Pandis N. 2013. Are clustering effects accounted for in statistical analysis in leading dental specialty journals? J Dent. 41(3):265-70.

Gargon E, Gorst SL, Harman NL, Smith V, Matvienko-Sikar K, Williamson PR. 2018. Choosing important health outcomes for comparative effectiveness research: 4th annual update to a systematic review of core outcome sets for research. PLOS ONE. 13(12):e0209869. doi:10.1371/journal.pone.0209869.

Gratsia S, Koletsi D, Fleming PS, Pandis N. 2019a. Statistical testing against baseline in orthodontic research: a meta-epidemiologic study. Eur J Orthod. 41(2):165-171.

Gratsia S, Koletsi D, Fleming PS, Pandis N. 2019b. A priori power considerations in orthodontic research: a 3 year meta-epidemiologic study. Eur J Orthod. doi:10.1093/ejo/cjz061.

Holden ACL, Spallek H. 2018. Looking Gift-horses in the Mouth: Gift-giving, Incentives and Conflict of Interest in the Dental Profession. J Law Med. 25(3):794-799. 
Hua Fang, Walsh T, Glenny A-M, Worthington H. 2016. Thirty percent of abstracts presented at dental conferences are published in full: a systematic review. J Clin Epidemiol. 75:16-28.

doi:10.1016/j.jclinepi.2016.01.029.

Hua F., Walsh T, Glenny A-M, Worthington H. 2016. Surveys on Reporting Guideline Usage in Dental Journals. J Dent Res. 95(11):1207-1213. doi:10.1177/0022034516657803.

Ioannidis JPA. 2016. Why Most Clinical Research Is Not Useful. PLoS Med. 13(6):e1002049. doi:10.1371/journal.pmed.1002049.

Ioannidis JPA, Greenland S, Hlatky MA, Khoury MJ, Macleod MR, Moher D, Schulz KF, Tibshirani R. 2014. Increasing value and reducing waste in research design, conduct, and analysis. Lancet. 383(9912):166175. doi:10.1016/S0140-6736(13)62227-8.

Kagereki E, Gakonyo J, Simila H. 2016. Significance bias: an empirical evaluation of the oral health literature. BMC Oral Health. 16(1):53.

Kim JSJ, Kim D-K, Hong SJ. 2011. Assessment of errors and misused statistics in dental research. Int Dent J. 61(3):163-7.

Kiriakou J, Pandis N, Fleming PS, Madianos P, Polychronopoulou A. 2013. Reporting quality of systematic review abstracts in leading oral implantology journals. J Dent. 41(12):1181-7.

Koletsi D, Fleming PS, Behrents RG, Lynch CD, Pandis N. 2017. The use of tailored subheadings was successful in enhancing compliance with CONSORT in a dental journal. J Dent. 67:66-71.

Koletsi D, Fleming PS, Seehra J, Bagos PG, Pandis N. 2014. Are sample sizes clear and justified in RCTs published in dental journals? PloS One. 9(1):e85949. doi:10.1371/journal.pone.0085949.

Koletsi D, Karagianni A, Pandis N, Makou M, Polychronopoulou A, Eliades T. 2009. Are studies reporting significant results more likely to be published? Am J Orthod Dentofacial Orthop. 136(5):632.e1-5; discussion 632-633. doi:10.1016/j.ajodo.2009.02.024.

Koletsi D, Madahar A, Fleming PS, Pandis N. 2015. Statistical testing against baseline was common in dental research. J Clin Epidemiol. 68(7):776-81.

Koletsi D, Pandis N, Fleming PS. 2014. Sample size in orthodontic randomized controlled trials: are numbers justified? Eur J Orthod. 36(1):67-73. doi:10.1093/ejo/cjt005.

Koletsi D, Pandis N, Polychronopoulou A, Eliades T. 2012a. Mislabeling controlled clinical trials (CCTs) as "randomized clinical trials (RCTs)" in dental specialty journals. J Evid-Based Dent Pract. 12(3):124-130. doi:10.1016/j.jebdp.2012.05.002.

Koletsi D, Pandis N, Polychronopoulou A, Eliades T. 2012b. What's in a title? An assessment of whether randomized controlled trial in a title means that it is one. Am J Orthod Dentofac Orthop Off Publ Am Assoc Orthod Its Const Soc Am Board Orthod. 141(6):679-685. doi:10.1016/j.ajodo.2011.12.020.

Koletsi D, Pandis N, Polychronopoulou A, Eliades T. 2012c. Does published orthodontic research account for clustering effects during statistical data analysis? Eur J Orthod. 34(3):287-92. 
Koufatzidou M, Koletsi D, Fleming PS, Polychronopoulou A, Pandis N. 2019. Outcome reporting discrepancies between trial entries and published final reports of orthodontic randomized controlled trials. Eur J Orthod. 41(3):225-230. doi:10.1093/ejo/cjy046.

Lee DJ. 2011. Research in prosthodontics: a 10-year observation of trends in topics, collaboration, and funding. Int J Prosthodont. 24(5):473-478.

Lee KC, Chuang S-K. 2020. Financial conflicts of interest in dentistry: how much money do providers receive from industry? Gen Dent. 68(1):56-60.

Lieber R, Pandis N, Faggion CM. 2020. Reporting and handling of incomplete outcome data in implant dentistry: A survey of randomized clinical trials. J Clin Periodontol. 47(2):257-266. doi:10.1111/jcpe.13222.

Livas C, Kouskoura T, Ren Y, Katsaros C, Pandis N. 2014. Are claims made in orthodontic journal advertisements evidence-supported? Angle Orthod. doi:10.2319/040814-258.1.

Livas C, Pandis N, Ren Y. 2014. Full-text publication of abstracts presented at European Orthodontic Society congresses. Eur J Orthod. 36(5):569-575. doi:10.1093/ejo/cjt089.

Madhavji A, Araujo EA, Kim KB, Buschang PH. 2011. Attitudes, awareness, and barriers toward evidencebased practice in orthodontics. Am J Orthod Dentofacial Orthop. 140(3):309-316.e2.

doi:10.1016/j.ajodo.2010.05.023.

Needleman I, Chin S, O'Brien T, Petrie A, Donos N. 2012. Systematic review of outcome measurements and reference group(s) to evaluate and compare implant success and failure. J Clin Periodontol. 39(s12):122-132. doi:10.1111/j.1600-051X.2011.01836.x.

Nieminen P, Sipilä K, Takkinen H-M, Renko M, Risteli L. 2007. Medical theses as part of the scientific training in basic medical and dental education: experiences from Finland. BMC Med Educ. 7(1):51. doi:10.1186/1472-6920-7-51.

Nuijten MB, Hartgerink CHJ, van Assen MALM, Epskamp S, Wicherts JM. 2016. The prevalence of statistical reporting errors in psychology (1985-2013). Behav Res Methods. 48(4):1205-1226. doi:10.3758/s13428-015-0664-2.

Ouzzani M, Hammady H, Fedorowicz Z, Elmagarmid A. 2016. Rayyan-a web and mobile app for systematic reviews. Syst Rev. 5(1):210. doi:10.1186/s13643-016-0384-4.

Pandis N, Polychronopoulou A, Eliades T. 2010. An assessment of quality characteristics of randomised control trials published in dental journals. J Dent. 38(9):713-21.

Pandis N, Polychronopoulou A, Madianos P, Makou M, Eliades T. 2011. Reporting of research quality characteristics of studies published in 6 major clinical dental specialty journals. J Evid-Based Dent Pract. 11(2):75-83. doi:10.1016/j.jebdp.2010.11.026.

Papageorgiou SN, Antonoglou GN, Martin C, Eliades T. 2019. Methods, transparency and reporting of clinical trials in orthodontics and periodontics. J Orthod. 46(2):101-109. 
Papageorgiou SN, Antonoglou GN, Sándor GK, Eliades T. 2017. Randomized clinical trials in orthodontics are rarely registered a priori and often published late or not at all. PloS One. 12(8):e0182785. doi:10.1371/journal.pone.0182785.

Papageorgiou SN, Kloukos D, Petridis H, Pandis N. 2015. An Assessment of the Risk of Bias in Randomized Controlled Trial Reports Published in Prosthodontic and Implant Dentistry Journals. Int J Prosthodont. 28(6):586-93.

Papageorgiou SN, Xavier GM, Cobourne MT, Eliades T. 2018. Registered trials report less beneficial treatment effects than unregistered ones: a meta-epidemiological study in orthodontics. J Clin Epidemiol. 100:44-52. doi:10.1016/j.jclinepi.2018.04.017.

Polychronopoulou A, Pandis N, Eliades T. 2010. Assessment of publication bias in dental specialty journals. J Evid-Based Dent Pract. 10(4):207-11.

Popelut A, Valet F, Fromentin O, Thomas A, Bouchard P. 2010. Relationship between sponsorship and failure rate of dental implants: a systematic approach. PloS One. 5(4):e10274.

Rice DB, Raffoul H, loannidis JPA, Moher D. 2020. Academic criteria for promotion and tenure in biomedical sciences faculties: cross sectional analysis of international sample of universities. BMJ. 369:m2081. doi:10.1136/bmj.m2081.

Rushing DC, Rushing CJ, Ospina A, McClure S, Cummings N. 2018. Publication Incidence of Abstracts Presented at the American Association of Oral and Maxillofacial Surgeons Meeting: 2010 to 2014. J Oral Maxillofac Surg Off J Am Assoc Oral Maxillofac Surg. 76(10):2051-2056. doi:10.1016/j.joms.2018.06.004.

Saltaji H, Armijo-Olivo S, Cummings GG, Amin M, Flores-Mir C. 2017. Randomized clinical trials in dentistry: Risks of bias, risks of random errors, reporting quality, and methodologic quality over the years 1955-2013. PloS One. 12(12):e0190089. doi:10.1371/journal.pone.0190089.

Sarkis-Onofre R, Poletto-Neto V, Cenci MS, Pereira-Cenci T, Moher D. 2017. Impact of the CONSORT Statement endorsement in the completeness of reporting of randomized clinical trials in restorative dentistry. J Dent. 58:54-59. doi:10.1016/j.jdent.2017.01.009.

Scholey JM, Harrison JE. 2005. Delay and failure to publish dental research. Evid Based Dent. 6(3):58-61. doi:10.1038/sj.ebd.6400347.

Smaïl-Faugeron V, Fron-Chabouis H, Courson F. 2014. Methodological quality and implications for practice of systematic Cochrane reviews in pediatric oral health: a critical assessment. BMC Oral Health. 14:35. doi:10.1186/1472-6831-14-35.

Smaïl-Faugeron V, Fron-Chabouis H, Durieux P. 2015. Clinical trial registration in oral health journals. J Dent Res. 94(3):8S-13S.

Spanou A, Koletsi D, Fleming PS, Polychronopoulou A, Pandis N. 2015. Statistical analysis in orthodontic journals: are we ignoring confounding? Eur J Orthod. doi:10.1093/ejo/cjv004.

Yuan JC-C, Shyamsunder N, Barao VAR, Lee DJ, Sukotjo C. 2011. Publication bias in five dental implant journals: an observation from 2005 to 2009. https://www.ncbi.nlm.nih.gov/pubmed/22010086. 
